\title{
The magnitude and clinical implications of the interobserver variability of echocardiographic left ventricular ejection fraction measurement
}

\section{OKarlo Golubić*, \\ Olvana Tomašić, Olvo Darko Gabrić}

University Hospital Centre "Sestre milosrdnice“, Zagreb, Croatia

\begin{abstract}
KEYWORDS: echocardiography, left ventricular ejection fraction, interobserver variability, heart failure. CITATION: Cardiol Croat. 2019;14(3-4):57. | https://doi.org/10.15836/ccar2019.57

*ADDRESS FOR CORRESPONDENCE: Karlo Golubić, Klinički bolnički centar Sestre milosrdnice, Vinogradska 29, HR-10000 Zagreb, Croatia. / Phone: +385-1-3787-111 / E-mail: karlo.golubic@gmail.com

ORCID: Karlo Golubić, https://orcid.org/0000-0003-0684-6333 • Ivana Tomašić, https://orcid.org/0000-0002-7345-5829 Ivo Darko Gabrić, https://orcid.org/0000-0003-4719-4634

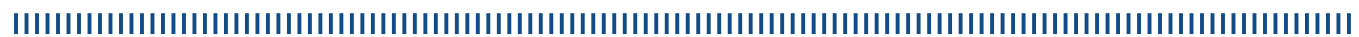

The ejection fraction (EF) is the most commonly used echocardiographic measure of the systolic left ventricular (LV) function. Echocardiography has however several limitations in measuring LV volumes and EF since the formulas for quantifications are based on geometrical assumptions, the EF parameters (end-systolic and end-diastolic volumes) are preload dependent and adequate projections are not always possible to obtain. Previous studies have also shown correlation between different echocardiographic techniques of obtaining EF and even correlation between echocardiography and other methods (MRI, SPECT, CT) but the data on interobserver and intraobserver variability remains scarce. ${ }^{1}$ In this study we examined the interobserver limits of agreement and the therapeutic implications of the uncertainty obtained by the result.
\end{abstract}

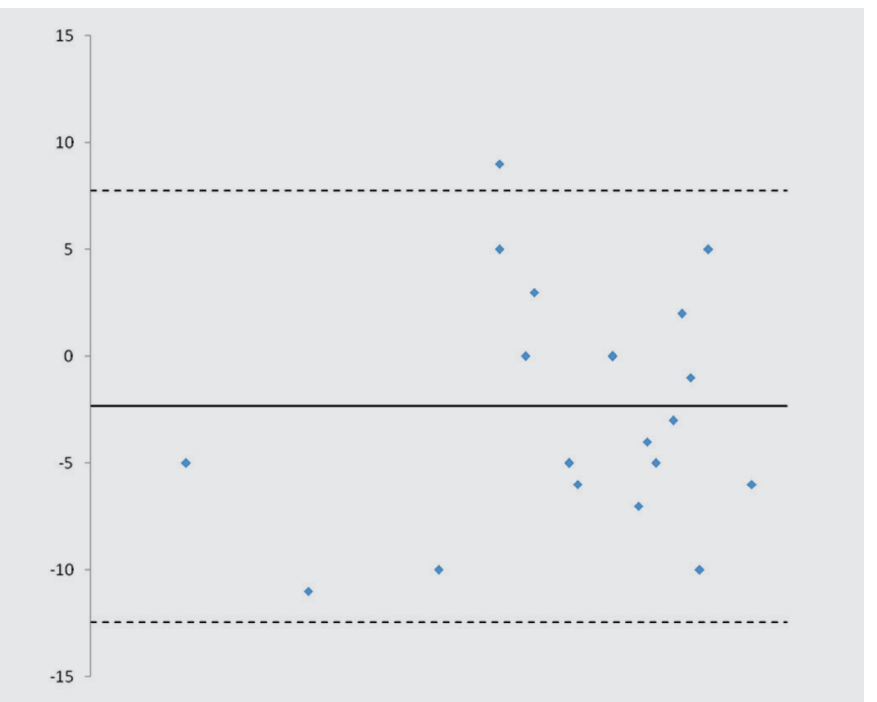

FIGURE 1. The Bland-Altman plot showing the limits of agreement. The limits of agreement are marked as dashed lines in the plot, specified as the average difference (bias) \pm 1.96 SD of the difference.
RECEIVED:

February 28, 2019

ACCEPTED:

March 24, 2019

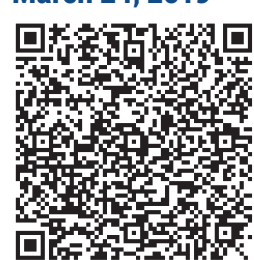

We examined 22 sequential patients referred to our echocardiography laboratory for LV function assessment. All echocardiography studies were performed by two independent cardiologists blinded to the measurements of the other observer. 2D methods including the biplane Simpson method and "eyeballing" were conducted, while automatic and semiautomatic 2D and 3D methods were not allowed. Bland-Altman analysis was performed to determine the systematic bias and limits of agreement (LOA) of EF between different observers (Figure 1). Next the LOA were used to obtain new "confidence intervals" on a previously gathered set of echocardiograms of 171 heart failure patients. We recorded the number of patients that now had different treatment recommendations according to the current ESC guidelines on heart failure and device therapy i.e. the "new" EF was $<35 \%$ or $>40 \%$. McNemar's test was used to determine the statistical significance of the difference with a two-tailed $\mathrm{P}=0.05$ taken as a threshold. The bias was calculated to be -2.3 with LOA (7.8 to -12.5) which is in agreement with similar studies. The number of patients that received "new" treatment recommendations was 99 for the lower LOA and 65 for upper LOA, $\mathrm{P}<0.001$ for both groups

We therefore conclude that the interobserver variability of the EF measurement can lead to significantly different treatment strategies in patients with heart failure. Echocardiographic methods introducing less variability (like automatic 3D techniques) should be preferred in determining the EF.

LITERATURE IIIIIIIIIIIIIIIIIIIIIIIIIIIIIIIIIIIIIIIIIIIIIIIIIIIIIIIIIIIIIIIIIIIIIIIIIIIIIIIIIIIIIIIIIIIIIIIIIIII

1. Thavendiranathan P, Grant AD, Negishi T, Plana JC, Popovic ZB, Marwick TH. Reproducibility of echocardiographic techniques for sequential assessment of left ventricular ejection fraction and volumes: application to patients undergoing cancer chemotherapy. J Am Coll Cardiol. 2013 Jan 8;61(1):77-84. https://doi.org/10.1016/j.jacc.2012.09.035 